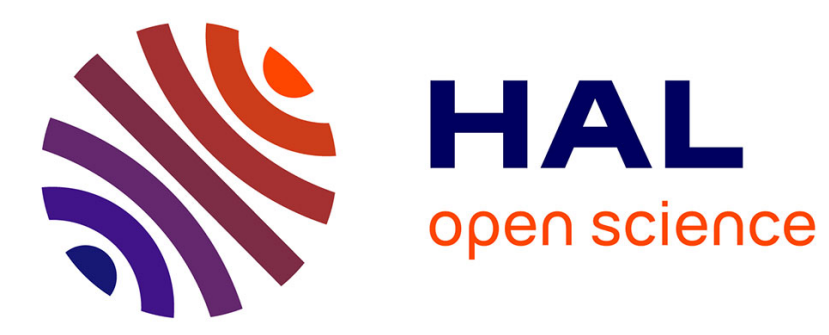

\title{
Target search and tracking using a fleet of UAVs in presence of decoys and obstacles
}

\author{
Julius Ibenthal, Luc Meyer, Michel Kieffer, Hélène Piet-Lahanier
}

\section{To cite this version:}

Julius Ibenthal, Luc Meyer, Michel Kieffer, Hélène Piet-Lahanier. Target search and tracking using a fleet of UAVs in presence of decoys and obstacles. 59th IEEE Conference on Decision and Control

(CDC), Dec 2020, Jeju (virtual), South Korea. 10.1109/CDC42340.2020.9303943 . hal-03206548

\section{HAL Id: hal-03206548 \\ https://hal.science/hal-03206548}

Submitted on 23 Apr 2021

HAL is a multi-disciplinary open access archive for the deposit and dissemination of scientific research documents, whether they are published or not. The documents may come from teaching and research institutions in France or abroad, or from public or private research centers.
L'archive ouverte pluridisciplinaire HAL, est destinée au dépôt et à la diffusion de documents scientifiques de niveau recherche, publiés ou non, émanant des établissements d'enseignement et de recherche français ou étrangers, des laboratoires publics ou privés. 


\title{
Target search and tracking using a fleet of UAVs in presence of decoys and obstacles
}

\author{
Julius Ibenthal, Luc Meyer, Hélène Piet-Lahanier, and Michel Kieffer
}

\begin{abstract}
This paper addresses the problem of searching and tracking of an a priori unknown number of indistinguishable targets spread over some geographical area using a fleet of UAVs. State perturbations and measurement noises are assumed to belong to bounded sets. In the monitored geographical area, some false targets (decoys) are present and may be erroneously considered as targets when observed under specific conditions. Moreover, obstacles in the search area constrain the displacements of the targets, alter the UAVs' trajectories, reduce their fields of view, and limit their communications. While the UAVs can detect targets or decoys when observation conditions are satisfied, they cannot identify them individually.

The search process relies on a robust bounded-error estimation approach which aim is to evaluate a set guaranteed to contain the actual states of already localized true targets and a set containing the states of targets still to be discovered. These two sets are used by each UAV to determine their control inputs in a distributed way to minimize future estimation uncertainty.

Simulations involving several UAVs illustrate that the proposed robust set-membership estimator and distributed control laws make it possible to efficiently search and track targets in the presence of decoys in a cluttered area.
\end{abstract}

\section{INTRODUCTION}

Searching and tracking mobile targets evolving in some geographical area is a demanding task that can be efficiently addressed by a fleet of UAVs. The search procedure often relies on the definition of a probability map of the presence of targets. This map is updated using observations collected by the UAVs. Their search trajectories are then evaluated to optimize the target state estimation accuracy. These trajectories can be obtained using explicit planning methods, which determine predicted environmental states, estimated target movements, and potential sensor observations (see, e.g., [16] for a review of path-constrained approaches). Determination of such UAVs trajectories has also been addressed through optimal control [5], [6] or derived from optimal strategies in a cooperative game approach [12].

For all the aforementioned methods, the performance of the resulting trajectories is tightly linked to the availability, quality, and reliability of the information collected by UAVs. In most cases, the quality of the measurements is assessed via probabilistic modeling. The measurement noise is usually represented using zero-mean Gaussian additive noise with a potentially varying variance, e.g., in [9]. Probabilities of false alarm and non-detection are used to represent corrupted

J. Ibenthal, L. Meyer and H. Piet-Lahanier are with Département Traitement de l'Information et Systèmes, ONERA, Univ. Paris Saclay, 91120 Palaiseau, France first_name. last_namedonera. fr

M. Kieffer is with Univ. Paris-Saclay, CNRS, CentraleSupélec, Laboratoire des signaux et systèmes, 91190, Gif-sur-Yvette, France michel.kieffer@l2s.centralesupelec.fr measurements. The former intends to represent the misinterpretation of objects as targets and the latter the absence of detection of targets located in the field of view [1], [8], [12]. As pointed out in [7], the a priori assumptions on the probability density functions (pdfs) describing the uncertainty and measurement noises may have a strong impact on the results of the search and track process. A potential alternative requiring less detailed assumptions consists of using a setmembership description of uncertainties, i.e., assuming that the target states remain within known bounded sets [4], [7]. Such an approach has been applied to cooperative guidance of a fleet of UAVs for target searching in [17], [18].

In this paper, we assume that each UAV is equipped with a sensor able to detect and localize targets in some compact subset of the search area. Contrary to [17] and [18], one considers here the presence of several false targets which could be erroneously interpreted as true targets under specific observation conditions. We also assume that the UAVs cannot identify the observed targets, i.e., they are not able to associate a specific measurement with a specific target. A robust distributed set-membership estimator is then proposed to determine subsets where the target states may be located and subsets which are guaranteed to contain no target state. The area of interest where the UAVs are performing the search may also contain obstacles. We propose a distributed control input design algorithm for the UAVs derived from the minimization of a criterion reflecting the expected estimation uncertainty and the potential risk of collision with obstacles.

The paper is organized as follows. Section II describes the multi-target multi-UAV localization problem, including the models of false targets and obstacles. Section III presents a distributed estimator that recursively provides sets containing the true targets. Section IV presents a control input design scheme to drive each UAV to minimize some measure of the expected estimation uncertainty. In Section V, the set-membership estimator and the control input design are evaluated on simulations. Section VI concludes this paper.

\section{PROBLEM FORMULATION}

Consider a fleet of $N_{\mathrm{u}}$ UAVs which aim is to search and track $N_{\mathrm{t}}$ potentially moving targets within some bounded geographical area, where $N_{\mathrm{t}}$ is constant but not known $a$ priori. This area may contain $N_{\mathrm{f}}$ potentially moving false target, where $N_{\mathrm{f}}$ is not necessarily constant (false targets may appear in the monitored area). A false target is erroneously interpreted as a true target when observed under specific conditions. The search area is cluttered with $N_{\mathrm{o}}$ a priori known static obstacles. 


\section{A. UAV and target state models}

Time is sampled with a constant period $T$. At time $k$ (time instant $t=k T), \mathbf{x}_{i, k}^{\mathrm{u}} \in \mathbb{R}^{n_{\mathrm{u}}}$ is the state vector of UAV $i$, $\mathbf{x}_{j, k}^{\mathrm{t}} \in \mathbb{R}^{n_{\mathrm{t}}}$ the state vector of target $j$, and $\mathbf{x}_{\ell, k}^{\mathrm{f}} \in \mathbb{R}^{n_{\mathrm{t}}}$ the state vector of false target $\ell$. The first three components of $\mathbf{x}_{j, k}^{\mathrm{t}}$ and $\mathbf{x}_{\ell, k}^{\mathrm{f}}$ are the spatial coordinates in a specific frame.

The dynamical models of the states of UAVs and of true and false targets are

$$
\begin{aligned}
& \mathbf{x}_{i, k+1}^{\mathrm{u}}=\mathbf{f}_{k}^{\mathrm{u}}\left(\mathbf{x}_{i, k}^{\mathrm{u}}, \mathbf{u}_{i, k}\right), \\
& \mathbf{x}_{j, k+1}^{\mathrm{t}}=\mathbf{f}_{k}^{\mathrm{t}}\left(\mathbf{x}_{j, k}^{\mathrm{t}}, \mathbf{v}_{j, k}^{\mathrm{t}}\right)
\end{aligned}
$$

and

$$
\mathbf{x}_{\ell, k+1}^{\mathrm{f}}=\mathbf{f}_{k}^{\mathrm{f}}\left(\mathbf{x}_{\ell, k}^{\mathrm{f}}, \mathbf{v}_{\ell, k}^{\mathrm{f}}\right),
$$

where $\mathbf{u}_{i, k}$ is the control input for UAV $i$, belonging to a set $\mathbb{U}$ of admissible control inputs; $\mathbf{v}_{j, k}^{\mathrm{t}}$ is an unknown target state perturbation belonging to the known box $\left[\mathbf{v}^{\mathrm{t}}\right]$, and $\mathbf{v}_{\ell, k}^{\mathrm{f}}$ is an unknown false target state input. One assumes that $\mathbf{x}_{j, k}^{\mathrm{t}} \in \mathbb{X}_{0}$ for all $k \geq 0, \mathbb{X}_{0} \subset \mathbb{R}^{n_{\mathrm{t}}}$ being some a priori known compact set. Moreover, one assumes that $\mathbf{f}_{k}^{\mathrm{u}}$ and $\mathbf{f}_{k}^{\mathrm{t}}$ are known, while $\mathbf{f}_{k}^{\mathrm{f}}$ is unknown.

\section{B. Description of obstacles}

Each obstacle $\mathcal{O}_{m}$, with $m \in\left\{1, \ldots, N_{\mathrm{o}}\right\}$, is assumed to be a polyhedron $\mathcal{P}_{m}$ of $\mathbb{R}^{3}$. It is modeled as $\mathcal{O}_{m}=\mathcal{P}_{m} \times$ $\mathbb{R}^{n_{\mathrm{t}}-3}$ to be consistent with the target state space. The UAVs know the locations of all the obstacles. Targets cannot enter the obstacles. Thus, the search space where the targets are evolving is : $\mathbb{X}_{\mathrm{s}}=\mathbb{X}_{0} \backslash \bigcup_{m \in\left\{1, \ldots, N_{\mathrm{o}}\right\}} \mathcal{O}_{m}$.

\section{Measurements}

At each time step, each UAV observes with its sensor a subset of $\mathbb{X}_{0}$, named the Field-Of-View (FoV). The observed region, for a given value $\mathrm{x}_{i, k}^{\mathrm{u}}$, is defined as the intersection between the FoV and the visibility region of the UAV. This latter is defined as the set of points from which a line segment connecting the UAV's location to this point does not intersect any obstacle. This region is computed for polyhedral obstacles using the algorithm proposed in [2] and [17]. The observed region is denoted by $\mathbb{F}_{i}\left(\mathbf{x}_{i, k}^{\mathrm{u}}\right) \subset \mathbb{R}^{n_{\mathrm{t}}}$.

We assume here that a true target is always detected when $\mathbf{x}_{j, k}^{\mathrm{t}} \in \mathbb{F}_{i}\left(\mathbf{x}_{i, k}^{\mathrm{u}}\right)$ and consequently a measurement of $\mathbf{x}_{j, k}^{\mathrm{t}}$ is collected by the UAV. For a false target, if $\mathbf{x}_{j, k}^{\mathrm{f}} \in \mathbb{F}_{i}\left(\mathbf{x}_{i, k}^{\mathrm{u}}\right)$, a measurement is obtained only when some additional observation condition is satisfied, i.e.,

$$
\mathbf{x}_{\ell, k}^{\mathrm{f}} \in \mathbb{F}_{i}\left(\mathbf{x}_{i, k}^{\mathrm{u}}\right) \text { and } g_{i, \ell}\left(\mathbf{x}_{i, k}^{\mathrm{u}}, \mathbf{x}_{\ell, k}^{\mathrm{f}}\right) \geqslant 0
$$

The UAVs have no prior information on the structure of $g_{i, \ell}$. The constraint $g_{i, \ell}\left(\mathbf{x}_{i, k}^{\mathrm{u}}, \mathbf{x}_{\ell, k}^{\mathrm{f}}\right) \geqslant 0$ indicates, for example, that the $\ell$-th false target is confused with a true target only if it is observed from specific points of view belonging to some polyhedral cone whose apex coordinates are the first three components of $\mathbf{x}_{\ell, k}^{\mathrm{f}}$.

Fig. 1 illustrates the 2D projection of the search space, of the state of true targets $\mathbf{x}_{i, k}^{\mathrm{t}}$ (filled circles) and of false targets $\mathbf{x}_{\ell, k}^{\mathrm{f}}$ (empty circles), as well as the projections of the cones within which false targets are considered as true targets.

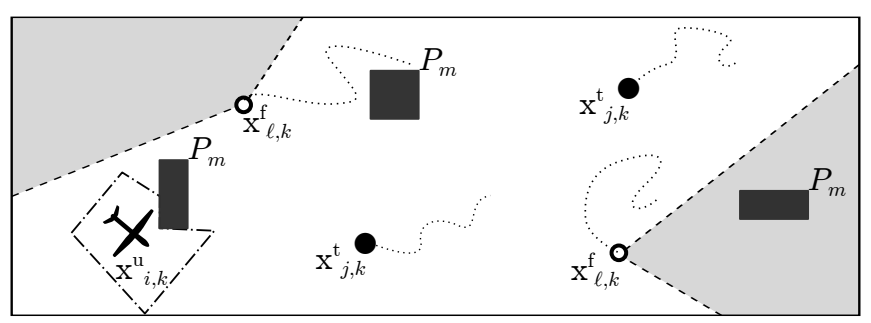

Fig. 1. 2D Projections $\left(x_{1}, x_{2}\right)$ of the search area, true targets $\mathbf{x}_{j, k}^{\mathrm{t}}$ (filled circles), false targets $\mathbf{x}_{\ell, k}^{\mathrm{f}}$ (empty circles), of their trajectories (dotted line), the UAV state $\mathbf{x}_{i, k}^{\mathrm{u}}$, UAV's FoV (dash-dotted line), and of the obstacles $\mathcal{O}_{m}$; The gray areas indicate the locations when $g_{i, \ell}\left(\mathbf{x}_{i, k}^{\mathrm{u}}, \mathbf{x}_{\ell, k}^{\mathrm{f}}\right) \geqslant 0$.

For each (true or false) target $j$ observed by UAV $i$ at time $k$, a noisy measurement $\mathbf{y}_{i, j, k} \in \mathbb{R}^{n_{y}}$ is obtained as

$$
\mathbf{y}_{i, j, k}=\mathbf{h}_{i}\left(\mathbf{x}_{i, k}^{\mathrm{u}}, \mathbf{x}_{j, k}^{\mathrm{t}, \mathrm{f}}\right)+\mathbf{w}_{i, j, k}
$$

where $\mathbf{h}_{i}$ is the observation equation of UAV $i$ and $\mathbf{w}_{i, j, k}$ is some measurement noise, bounded in a known box $\left[\mathbf{w}_{k}\right]$.

Contrary to [10], (5) only provides some information about the location of a true or false target, without any indication on the target identity. When several target states belong to $\mathbb{F}_{i}\left(\mathrm{x}_{i, k}^{\mathrm{u}}\right)$, as many distinct measurements are obtained. The set of measurements collected by UAV $i$ at time $k$ is thus

$$
\begin{aligned}
\mathcal{Y}_{i, k}= & \left\{\mathbf{y}_{i, j, k} \mid \mathbf{x}_{j, k}^{\mathrm{t}} \in \mathbb{F}_{i}\left(\mathbf{x}_{i, k}^{\mathrm{u}}\right), j=1, \ldots, N_{\mathrm{t}}\right\} \\
& \cup\left\{\mathbf{y}_{i, \ell, k} \mid \mathbf{x}_{\ell, k}^{\mathrm{f}} \in \mathbb{F}_{i}\left(\mathbf{x}_{i, k}^{\mathrm{u}}\right)\right. \\
& \left.\wedge g_{i, \ell}\left(\mathbf{x}_{i, k}^{\mathrm{u}}, \mathbf{x}_{\ell, k}^{\mathrm{f}}\right) \geqslant 0, \ell=1, \ldots, N_{\mathrm{f}}\right\} .
\end{aligned}
$$

The set containing all noise-free measurements related to true and false targets in the FoV is derived from $\mathcal{Y}_{i, k}$ by accounting for the bounded measurement noise $\left[\mathbf{w}_{k}\right]$, as

$$
\mathbb{Y}_{i, k}=\left\{\mathbf{y}-\left[\mathbf{w}_{k}\right] \mid \mathbf{y} \in \mathcal{Y}_{i, k}\right\}
$$

\section{Communications}

When UAVs $i$ and $j$ are in communication range, and their relative line of sight doesn't intersect any obstacle, both UAVs are able to exchange information. The ability to exchange information between UAVs at time $k$ is described by an undirected graph $\mathcal{G}_{k}=\left(\mathcal{N}_{\mathrm{u}}, \mathcal{E}_{k}\right) . \mathcal{N}_{\mathrm{u}}=\left\{1,2, \ldots, N_{\mathrm{u}}\right\}$ is the set of nodes (UAVs) and $\mathcal{E}_{k} \subset \mathcal{N}_{\mathrm{u}} \times \mathcal{N}_{\mathrm{u}}$ the set of edges of the network at time $k$, where $(i, j) \in \mathcal{E}_{k}$ indicates that UAVs $i$ and $j$ are able to communicate (without delay and without error) at time $k$. The set of neighbors of UAV $i$ at time $k$ is $\mathcal{N}_{i, k}=\left\{j \in \mathcal{N}_{\mathrm{u}} \mid(i, j) \in \mathcal{E}_{k}, i \neq j\right\}$.

\section{Set estimates fOR A Given UAV}

In the proposed approach, at time $k$, each UAV $i$ maintains a set-membership state estimate $\mathbb{X}_{i, k} \subset \mathbb{X}_{0}$, that has to contain all possible values of $\mathbf{x}_{j, k}^{\mathrm{t}}$, consistent with the information available to UAV $i$ up to time $k$. Due to the presence of false targets, the set $\mathbb{X}_{i, k}$ may also contain $\mathbf{x}_{\ell, k}^{\mathrm{f}}$ for some $\ell=1, \ldots, N_{\mathrm{f}}$. UAV $i$ also maintains a set $\overline{\mathbb{X}}_{i, k} \subset \mathbb{X}_{0}$ containing the possible state values of true targets not yet detected.

In what follows, we consider the evolution with time of the sets $\mathbb{X}_{i, k}$ and $\overline{\mathbb{X}}_{i, k}$ evaluated by a given UAV $i$. At each time 
step, as in the distributed Kalman filter [15], a prediction step is first performed, then measurements acquired by UAV $i$ are taken into account in a correction step, and finally, information coming from neighboring UAVs are processed in an information fusion step. Contrary to the Kalman filter set estimates are performed, initialized as $\mathbb{X}_{i, 0}=\emptyset$ and $\overline{\mathbb{X}}_{i, 0}=\mathbb{X}_{\mathrm{s}}$ for $i=1, \ldots, N_{\mathrm{u}}$.

\section{A. Prediction step}

Assume that at time $k$, UAV $i$ has evaluated $\mathbb{X}_{i, k}$ and $\overline{\mathbb{X}}_{i, k}$. At time $k+1$, UAV $i$ is able to evaluate the set of possible target state values that are consistent with $\mathbb{X}_{i, k}$, the true target dynamics (2), and the bounded state perturbation

$$
\mathbb{X}_{i, k+1 \mid k}=\mathbf{f}_{k}^{\mathrm{t}}\left(\mathbb{X}_{i, k},\left[\mathbf{v}_{k}\right]\right) \cap \mathbb{X}_{\mathrm{s}}
$$

Similarly, the predicted set $\overline{\mathbb{X}}_{i, k+1 \mid k}$ has to contain all possible state values of potentially undetected targets. Since all targets have been assumed to evolve according to the same dynamics (2), it is evaluated as

$$
\overline{\mathbb{X}}_{i, k+1 \mid k}=\mathbf{f}_{k}^{\mathrm{t}}\left(\overline{\mathbb{X}}_{i, k},\left[\mathbf{v}_{k}\right]\right) \cap \mathbb{X}_{\mathrm{s}}
$$

The presence of obstacles is taken into account in the prediction step as the UAVs are assumed to know their locations a priori. On the contrary, the false target dynamics are not taken into account. If a false target does not satisfy (8), then it might not belong to the predicted state set $\mathbb{X}_{i, k+1 \mid k}$. This will result in an improved differentiation between false and true targets. Furthermore, if a false target satisfies (8), then it will remain within the predicted set and only further measurements will allow to discriminate it.

\section{B. Correction step from measurements}

Assume that at time $k+1$, UAV $i$ collects the observations $\mathbb{F}_{i}\left(\mathbf{x}_{i, k+1}^{\mathrm{u}}\right)$ and obtains the set $\mathbb{Y}_{i, k+1}$ introduced in (7). To evaluate $\mathbb{X}_{i, k+1 \mid k+1}$, UAV $i$ has to account for the set $\mathbb{Y}_{i, k+1}$ containing all noise-free measurements as well as $\mathbb{X}_{i, k+1 \mid k}$ and $\overline{\mathbb{X}}_{i, k+1 \mid k} \cdot \mathbb{X}_{i, k+1 \mid k+1}$ is obtained as the union of three subsets. First, one has to consider the states of already detected targets (their states are in $\mathbb{X}_{i, k+1 \mid k}$ ), which have been observed at time $k+1$ (their states satisfy $\left.\mathbf{h}_{k+1}\left(\mathbf{x}_{i, k+1}^{\mathrm{u}}, \mathbf{x}\right) \in \mathbb{Y}_{i, k+1}\right)$. The states of such targets belong thus to

$$
\mathbb{S}_{1}=\left\{\mathbf{x} \in \mathbb{X}_{i, k+1 \mid k} \mid \mathbf{h}_{k+1}\left(\mathbf{x}_{i, k+1}^{\mathrm{u}}, \mathbf{x}\right) \in \mathbb{Y}_{i, k+1}\right\}
$$

Second, one has to consider the states of not yet detected targets (their states are in $\overline{\mathbb{X}}_{i, k+1 \mid k}$ ), which have been observed at time $k+1$ (their states satisfy $\left.\mathbf{h}_{k+1}\left(\mathbf{x}_{i, k+1}^{\mathrm{u}}, \mathbf{x}\right) \in \mathbb{Y}_{i, k+1}\right)$. The states of such targets belong to

$$
\mathbb{S}_{2}=\left\{\mathbf{x} \in \overline{\mathbb{X}}_{i, k+1 \mid k} \mid \mathbf{h}_{k+1}\left(\mathbf{x}_{i, k+1}^{\mathrm{u}}, \mathbf{x}\right) \in \mathbb{Y}_{i, k+1}\right\}
$$

Third, for some parts of $\mathbb{X}_{i, k+1 \mid k}$, no information is available at time $k+1$ (the part outside $\mathbb{F}_{i}\left(\mathbf{x}_{i, k+1}^{\mathrm{u}}\right)$ ). Thus, the set

$$
\mathbb{S}_{3}=\mathbb{X}_{i, k+1 \mid k} \backslash \mathbb{F}_{i}\left(\mathbf{x}_{i, k+1}^{\mathrm{u}}\right)
$$

where $\mathbb{B} \backslash \mathbb{A}=\{x \in \mathbb{B} \mid x \notin \mathbb{A}\}$ has also to belong to $\mathbb{X}_{i, k+1 \mid k+1}$. To summarize, one has

$$
\mathbb{X}_{i, k+1 \mid k+1}=\mathbb{S}_{1} \cup \mathbb{S}_{2} \cup \mathbb{S}_{3}
$$
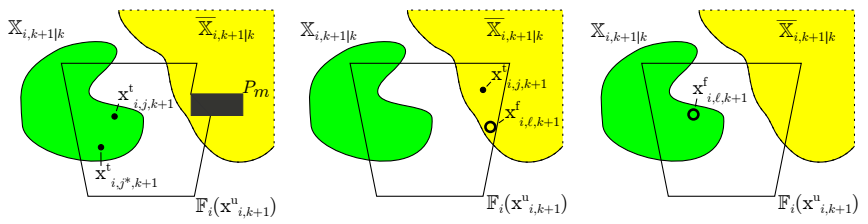

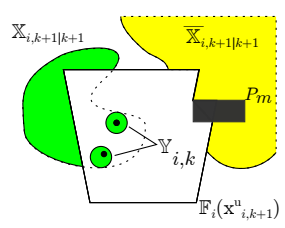

(a)

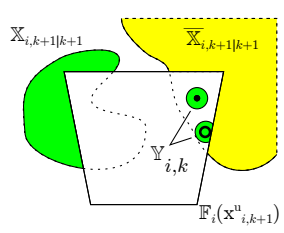

(b)

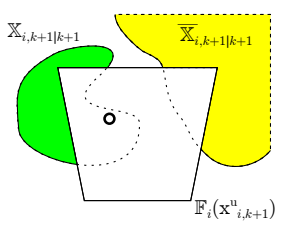

(c)
Fig. 2. Description of $\mathbb{X}_{i, k+1 \mid k}$ and $\overline{\mathbb{X}}_{i, k+1 \mid k}$ (top) and of $\mathbb{X}_{i, k+1 \mid k+1}$ and $\overline{\mathbb{X}}_{i, k+1 \mid k+1}$ (bottom); (a) the measurement corresponds to two previously detected target $\left(j\right.$ and $\left.j^{*}\right) ;(b)$ the measurement corresponds to new false and true targets ((4) is satisfied); (c) a previously detected false target is observed in $\mathbb{F}_{i}\left(\mathbf{x}_{i, k+1}^{\mathrm{u}}\right)$ but (4) is not satisfied.

Once all measurements obtained within the FoV $\mathbb{F}_{i}\left(\mathbf{x}_{i, k+1}^{\mathrm{u}}\right)$ have been processed, the set containing all possible state values of true targets not yet detected can be updated as

$$
\overline{\mathbb{X}}_{i, k+1 \mid k+1}=\overline{\mathbb{X}}_{i, k+1 \mid k} \backslash \mathbb{F}_{i}\left(\mathbf{x}_{i, k+1}^{\mathrm{u}}\right) .
$$

Then UAV $i$ is able to evaluate the set

$$
\widetilde{\mathbb{X}}_{i, k+1 \mid k+1}=\mathbb{X}_{\mathrm{s}} \backslash\left(\mathbb{X}_{i, k+1 \mid k+1} \cup \overline{\mathbb{X}}_{i, k+1 \mid k+1}\right),
$$

guaranteed not to contain the states of any true target. Fig. 2 illustrates the correction step from measurements.

\section{Fusion step after communications}

UAV $i$ exchanges the sets $\mathbb{X}_{i, k+1 \mid k+1}$ and $\overline{\mathbb{X}}_{i, k+1 \mid k+1}$ with all its neighbors $\mathcal{N}_{i, k+1}$ in communication vicinity, as defined in subsection II-D, at the end of each time step $k+1$.

UAV $i$ evaluates the information from its neighbors as the union of the sets $\mathbb{X}_{\ell, k+1 \mid k+1}$ containing all the possible state values, deprived from the union of all sets $\widetilde{\mathbb{X}}_{\ell, k+1 \mid k+1}$ which have been proved not to contain a target at time $k+1$

$$
\mathbb{X}_{i, k+1}=\bigcup_{\ell \in \overline{\mathcal{N}}_{i, k+1}} \mathbb{X}_{\ell, k+1 \mid k+1} \backslash \bigcup_{\ell \in \overline{\mathcal{N}}_{i, k+1}} \widetilde{\mathbb{X}}_{\ell, k+1 \mid k+1},
$$

where $\overline{\mathcal{N}}_{i, k+1}=\mathcal{N}_{i, k+1} \cup\{i\}$. The subset of the state space still to be explored is the intersection of the unexplored parts of the state space by UAV $i$ and those of its neighbors

$$
\overline{\mathbb{X}}_{i, k+1}=\bigcap_{\ell \in \overline{\mathcal{N}}_{i, k+1}} \overline{\mathbb{X}}_{\ell, k+1 \mid k+1}
$$

Fig. 3 illustrates the fusion step after communications resulting from (15) and (16). The figure highlights the fact that information on target locations is not differentiated for each target, since they are collected in a single set $\mathbb{X}_{i, k+1}$. As the information is defined as a whole, it makes it more difficult to differentiate between false and true targets $(*)$. 


\section{UAV 1}
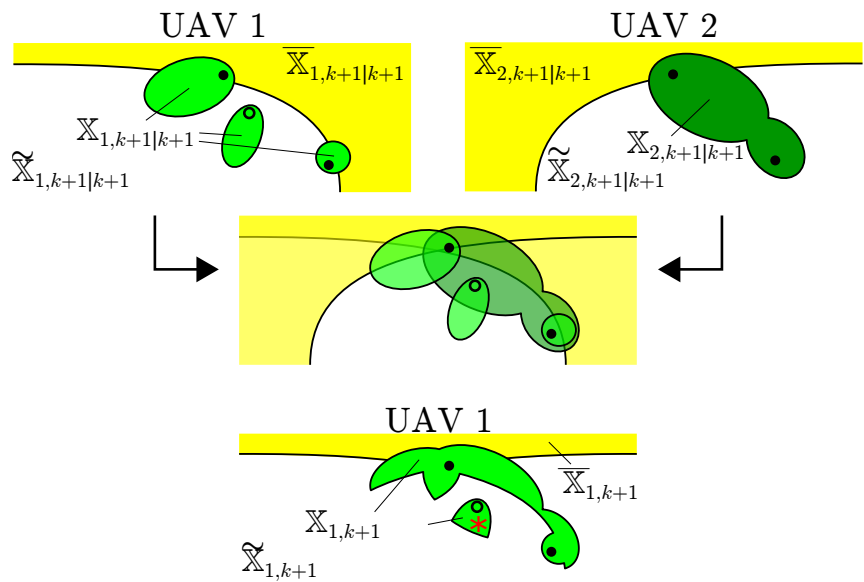

Fig. 3. Evaluation of the state estimates by UAV 1 after receiving Information from UAV 2: $\mathbb{X}_{1, k+1 \mid k+1}$ and $\mathbb{X}_{1, k+1}$ are in light green whereas $\mathbb{X}_{2, k+1 \mid k+1}$ in dark green.

\section{COOPERATIVE CONTROL DESIGN}

At time $k$, after processing the information from measurements or communication, each UAV $i$ has access to $\mathbb{X}_{i, k}$, and $\overline{\mathbb{X}}_{i, k}$ and is able to evaluate the state estimation uncertainty

$$
\Phi_{i}\left(\mathbb{X}_{i, k}, \overline{\mathbb{X}}_{i, k}\right)=\phi\left(\mathbb{X}_{i, k}\right)+\phi\left(\overline{\mathbb{X}}_{i, k}\right)
$$

where $\phi(\mathbb{X})$ is the measure of a set $\mathbb{X}, \phi\left(\mathbb{X}_{i, k}\right)$ is the state estimation uncertainty of detected targets, and $\phi\left(\overline{\mathbb{X}}_{i, k}\right)$ of not yet detected targets. Ideally, the UAVs should then determine, in a distributed way, the sequence of control inputs that will reduce (17) as fast as possible.

In this paper, one considers some time horizon $h>0$ and determines, at each time instant $k$, the sequence of control inputs which minimize the average value of (17) at time $k+h$

$$
\Phi_{k+h}=\frac{1}{N_{\mathrm{u}}} \sum_{i=1}^{N_{\mathrm{u}}} \Phi_{i}\left(\mathbb{X}_{i, k+h}, \overline{\mathbb{X}}_{i, k+h}\right) .
$$

One relies on the distributed Model Predictive Control (MPC) formalism introduced, e.g., in [3], [14] and considers several additional simplifications to enable a distributed evaluation of the control inputs.

\section{A. Evaluation of the control input}

A suboptimal sequential approach is proposed to evaluate, for each UAV, a sequence of control inputs minimizing (18). Consider UAV $i$ and assume that a subset $\mathcal{N}_{i, k}^{\mathrm{P}} \subset \mathcal{N}_{i, k}$ of its neighbors have already evaluated and transmitted their own control inputs $\left\{\widehat{\mathbf{u}}_{\ell, k+1}, \ldots, \widehat{\mathbf{u}}_{\ell, k+h}\right\}_{\ell \in \mathcal{N}_{i, k}^{\mathrm{P}}}$ as well as their state $\mathbf{x}_{i, k}^{\mathrm{u}}$ at time $k$ to $\mathrm{UAV} i$.

At time $k$, UAV $i$ has to determine the sequence of control inputs $\mathbf{u}_{i, k+1: h}=\left\{\mathbf{u}_{i, k+1}, \ldots, \mathbf{u}_{i, k+h}\right\}$ that minimizes $\Phi\left(\mathbb{X}_{i, k+h}, \overline{\mathbb{X}}_{i, k+h}\right)$. This has to be performed accounting for the control inputs $\mathcal{U}_{i, k}=\left\{\widehat{\mathbf{u}}_{\ell, k+1}, \ldots, \widehat{\mathbf{u}}_{\ell, k+h}\right\}_{\ell \in \mathcal{N}_{i, k}^{\mathrm{P}}}$ evaluated by a subset of its neighbors.

In practice, due to the short time horizon $h$, minimizing only $\Phi\left(\mathbb{X}_{i, k+h}, \overline{\mathbb{X}}_{i, k+h}\right)$ may lead to deadlocks. Conse- quently, UAV $i$ considers the regularized cost function

$$
\begin{aligned}
J\left(\mathbf{u}_{i, k+1: h}, \mathcal{U}_{i, k}\right)= & J^{\mathrm{m}}\left(\mathbf{u}_{i, k+1: h}, \mathcal{U}_{i, k}\right)+\alpha^{\mathrm{d}} J^{\mathrm{d}}\left(\mathbf{u}_{i, k+1: h}\right) \\
& +\alpha^{\mathrm{s}} J^{\mathrm{s}}\left(\mathbf{u}_{i, k+1: h}\right) .
\end{aligned}
$$

to be minimized with respect to $\mathbf{u}_{i, k+1: h}$. In (19), $J^{\mathrm{m}}\left(\mathbf{u}_{i, k+1: h}, \mathcal{U}_{i, k}\right)$ represents the state estimation uncertainty (17) at time $k+h, J^{\mathrm{d}}\left(\mathbf{u}_{i, k+1: h}\right)$ represents the distance between UAV $i$ and remote components of the set-membership state estimates, and $J^{\mathrm{s}}\left(\mathbf{u}_{i, k+1: h}\right)$ evaluates a risk of collision between UAV $i$ and the obstacles. The constants $\alpha^{\mathrm{d}}$ and $\alpha^{\mathrm{s}}$ adjust the relative importance of the terms in (19), which are detailed in the following sections.

UAV $i$ determines the sequence of control input $\widehat{\mathbf{u}}_{i, k+1: h}$ that minimizes (19) by gradient search starting from the sequence of control inputs $\widehat{\mathbf{u}}_{i, k+0: h-1}$ evaluated by UAV $i$ at time $k-1$.

1) State estimation uncertainty: The first term in (19) is the predicted state estimation uncertainty evaluated at time $k+h$

$$
\begin{aligned}
J^{\mathrm{m}}\left(\mathbf{u}_{i, k+1: h}, \mathcal{U}_{i, k}\right)= & \phi\left(\mathbb{X}_{i, k+h}^{\mathrm{P}}\left(\mathbf{u}_{i, k+1: h}, \mathcal{U}_{i, k}\right)\right) \\
& +\alpha^{\mathrm{m}} \phi\left(\overline{\mathbb{X}}_{i, k+h}^{\mathrm{P}}\left(\mathbf{u}_{i, k+1: h}, \mathcal{U}_{i, k}\right)\right),
\end{aligned}
$$

where $\mathbb{X}_{i, k+h}^{\mathrm{P}}\left(\mathbf{u}_{i, k+1: h}, \mathcal{U}_{i, k}\right)$ and $\overline{\mathbb{X}}_{i, k+h}^{\mathrm{P}}\left(\mathbf{u}_{i, k+1: h}, \mathcal{U}_{i, k}\right)$ are predicted values of $\mathbb{X}_{i, k+h}$ and $\overline{\mathbb{X}}_{i, k+h}$ evaluated from $\mathbb{X}_{i, k}$ and $\overline{\mathbb{X}}_{i, k}$ using $\mathbf{u}_{i, k+1: h}$ and $\mathcal{U}_{i, k}$. The parameter $\alpha^{\mathrm{m}}$ adjusts the relative importance of each term of (20).

To evaluate $\mathbb{X}_{i, k+h}^{\mathrm{P}}\left(\mathbf{u}_{i, k+1: h}, \mathcal{U}_{i, k}\right)$ and $\overline{\mathbb{X}}_{i, k+h}^{\mathrm{P}}\left(\mathbf{u}_{i, k+1: h}\right.$, $\left.\mathcal{U}_{i, k}\right)$, several hypotheses are considered. One assumes that the set of neighbors $\mathcal{N}_{i, k}^{\mathrm{P}}$ of UAV $i$ does not change between time $k$ and $k+h$, and that estimates for all the UAVs in $\mathcal{N}^{\mathrm{u}}$ are equal at time $k$, i.e, $\mathbb{X}_{\ell, k}=\mathbb{X}_{i, k}$ and $\overline{\mathbb{X}}_{\ell, k}=$ $\overline{\mathbb{X}}_{i, k}, \ell \in \mathcal{N}_{i, k}^{P}$. Moreover, since UAV $i$ has only access to the control inputs evaluated by its neighbors in $\mathcal{N}_{i, k}^{\mathrm{P}}$, only the information that will be provided by these agents via communication at the time steps $k+\kappa, \kappa=1, \ldots, h$, will be considered in the construction of $\mathbb{X}_{i, k+h}^{\mathrm{P}}\left(\mathbf{u}_{i, k+1: h}, \mathcal{U}_{i, k}\right)$ and $\overline{\mathbb{X}}_{i, k+h}^{\mathrm{P}}\left(\mathbf{u}_{i, k+1: h}, \mathcal{U}_{i, k}\right)$. UAV $i$ will further neglect all information that its neighbors may receive from their own neighbors, which do not belong to $\mathcal{N}_{i, k}^{\mathrm{P}}$. Using these assumptions, the approach described in [10] is employed to evaluate $\mathbb{X}_{i, k+h}^{\mathrm{P}}\left(\mathbf{u}_{i, k+1: h}, \mathcal{U}_{i, k}\right)$ and $\overline{\mathbb{X}}_{i, k+h}^{\mathrm{P}}\left(\mathbf{u}_{i, k+1: h}, \mathcal{U}_{i, k}\right)$. In this approach, the prediction steps employ (8) and (9). In the correction steps, (14) is employed as is, however, all potentially newly discovered targets are ignored in (13), by considering only $\mathbb{S}_{3}$. Finally, in the information fusion steps, (15) and (16) are also employed as is.

2) Distance to the sets: Assume that $\mathbb{X}_{i, k}$ consists of $N_{i, k}$ disjoint components

$$
\mathbb{X}_{i, k}=\bigcup_{\ell=1}^{N_{i, k}} \mathbb{X}_{i, \ell, k}
$$

and let $j$ denote the component minimizing the weighted Hausdorff distance to $\mathrm{x}_{i, k}^{\mathrm{u}}$, i.e.,

$$
j=\arg \min _{\ell} \phi\left(\mathbb{X}_{i, \ell, k}\right) d\left(\mathbf{x}_{i, k}^{\mathrm{u}}, \mathbb{X}_{i, \ell, k}\right),
$$


where $d\left(\mathbf{x}_{i, k}^{\mathrm{u}}, \mathbb{X}_{i, \ell, k}\right)$ is the Hausdorff distance between $\mathbf{x}_{i, k}^{\mathrm{u}}$ and $\mathbb{X}_{i, \ell, k}$. When (20) remains constant for all $\mathbf{u}_{i, k+1: h}$, it may be useful to drive UAV $i$ towards the closest set estimate with a large volume. This is obtained considering the term

$$
\begin{aligned}
J^{\mathrm{d}}\left(\mathbf{u}_{i, k+1: h}\right) & =\phi\left(\mathbb{X}_{i, j, k}\right) d\left(\mathbb{X}_{i, j, k}, \mathbf{x}_{i, k+h}^{\mathrm{u}, \mathrm{P}}\left(\mathbf{u}_{i, k+1: h}\right)\right) \\
& +\phi\left(\overline{\mathbb{X}}_{i, k}\right) d\left(\overline{\mathbb{X}}_{i, k}, \mathbf{x}_{i, k+h}^{\mathrm{u}, \mathrm{P}}\left(\mathbf{u}_{i, k+1: h}\right)\right)
\end{aligned}
$$

where $\mathbf{x}_{i, k+h}^{\mathrm{u}, \mathrm{P}}\left(\mathbf{u}_{i, k+1: h}\right)$ is the predicted value of $\mathbf{x}_{i, k+h}^{\mathrm{u}}$ when the sequence of control inputs of UAV $i$ is $\mathbf{u}_{i, k+1: h}$. One only minimizes the distance corresponding to the position of the UAV obtained at time $k+h . J^{\mathrm{d}}\left(\mathbf{u}_{i, k+1: h}\right)$ does not account for the control input of other UAVs.

3) Distance to obstacles: This criterion is designed to represent the risk of collision between UAVs and obstacles. It considers that a collision may occur when the distance between a UAV and the boundary of a polytope is less than some threshold $d^{\mathrm{col}}$. The criterion expression has been designed in order to satisfy the following properties. Its amplitude of variations should be very small when the UAV is located far from the obstacle, and increase very fast in the vicinity of an obstacle. The function should be smooth to be consistent with gradient search. A suitable candidate for satisfying these properties, as presented in [19], is the following risk expression:

$$
\begin{aligned}
& J^{\mathrm{s}}\left(\mathbf{u}_{i, k+1: h}\right)= \\
& \quad \frac{1}{2}\left[1-\tanh \left(\frac{d\left(\mathcal{O}_{m}, \mathbf{x}_{i, k+h}^{\mathrm{u}, \mathrm{P}}\left(\mathbf{u}_{i, k+1: h}\right)\right)-\alpha^{\mathrm{s} 1}}{\alpha^{\mathrm{s} 2}}\right)\right],
\end{aligned}
$$

where $\alpha^{\mathrm{s} 1}$ and $\alpha^{\mathrm{s} 2}$ are used to tune the shape of the function which varies between 0 (no risk of collision) and 1 (collision will occur). $\alpha^{\mathrm{s} 2}$ defines the width of the region where the criterion variations are fast and $\alpha^{\mathrm{s} 1}$ defines the center of this region and is related to $d^{\text {col }}$ by $\alpha^{\mathrm{s} 1}-\alpha^{\mathrm{s} 2}>=d^{\text {col }}$.

\section{B. Practical issues}

In practice, the order in which the UAVs compute their control input at each time step $k$ may be optimized. Possible suboptimal approaches consist of starting with the most connected UAV and continue with its neighbors or start with the UAV of the smallest index and continue with the connected UAVs. This strategy is similar to that considered in the binary log-linear learning algorithm [13]. If the graph $\mathcal{G}_{k}$ consists of several disconnected components, the evaluation of the control input has to be started selecting one UAV per connected component.

The first UAV evaluates its control input independently of the other UAVs. Assume without loss of generality that its index is $i=1$. UAV 1 determines the vector

$$
\widehat{\mathbf{u}}_{1, k+1: h}=\arg \min _{\mathbf{u}_{1, k+1: h}} J\left(\mathbf{u}_{1, k+1: h}\right),
$$

where the minimization of $J\left(\mathbf{u}_{1, k+1: h}\right)$ defined in (19) is over all $\mathbf{u}_{1, k+1: h} \in \mathbb{U}^{h}$, without accounting for the presence of its neighbors. Then UAV $\ell$, connected to UAV 1, will determine $\mathbf{u}_{\ell, k+1: h} \in \mathbb{U}^{h}$ minimizing $J\left(\mathbf{u}_{\ell, k+1: h}, \widehat{\mathbf{u}}_{1, k+1: h}\right)$, accounting for the information provided by UAV 1 controlled using $\widehat{\mathbf{u}}_{1, k+1: h}$.

To further simplify the search, one may assume in the MPC approach that, at time $k$, only the control input at time $k+1$ is evaluated and that the control inputs at the next time instants remain constant. This simplification has been considered in the following simulations.

The complexity of the proposed approach is independent of the number of targets since a single set is used by each UAV to represent the possible states of all targets. Moreover the complexity is linear with the number of UAVs thanks to the sequential evaluation of the control input.

\section{Simulations}

We consider the search of $N_{\mathrm{t}}=2$ true ground targets assumed to move in a plane. The sampling period is $T=1 \mathrm{~s}$. The coordinates at time $k$ of target $j$ in some reference frame attached to the plane are $\left(x_{j, k+1,1}^{\mathrm{t}}, x_{j, k+1,2}^{\mathrm{t}}\right)^{\mathrm{\top}}$. The targets move with a constant speed $x_{j, k, 3}^{\mathrm{t}}=5 \mathrm{~km} / \mathrm{h}$. The heading angle $x_{j, k, 4}^{\mathrm{t}}$ is controlled with an input $v_{j, k}$ uniformly distributed in the interval $[-\pi / 5, \pi / 5]$ and changing at each time instant. The target state vector $\mathbf{x}_{j, k}^{\mathrm{t}}$ evolves according to

$$
\left(\begin{array}{c}
x_{j, k+1,1}^{\mathrm{t}} \\
x_{j, k+1,2}^{\mathrm{t}} \\
x_{j, k+1,3}^{\mathrm{t}} \\
x_{j, k+1,4}^{\mathrm{t}}
\end{array}\right)=\left(\begin{array}{c}
x_{j, k, 1}^{\mathrm{t}}+T x_{j, k, 4}^{\mathrm{t}} \cos \left(x_{j, k, 4}^{\mathrm{t}}+v_{j, k}\right) \\
x_{j, k, 2}^{\mathrm{t}}+T x_{j, k, 4}^{\mathrm{t}} \sin \left(x_{j, k, 4}^{\mathrm{t}}+v_{j, k}\right) \\
x_{j, k, 3}^{\mathrm{t}} \\
x_{j, k, 4}^{\mathrm{t}}+v_{j, k}
\end{array}\right) .
$$

The search is performed by $N_{\mathrm{u}}=4 \mathrm{UAVs}$ which spatial coordinates at time $k$ are $\left(x_{i, k, 1}^{\mathrm{u}}, x_{i, k, 2}^{\mathrm{u}}, x_{i, k, 3}^{\mathrm{u}}\right)^{\top}, i=$ $1, \ldots, N_{\mathrm{u}}$. The initial altitude $x_{i, 0,3}^{\mathrm{u}}$ is $100 \mathrm{~m}$ and remains constant. The norm of the speed $x_{i, k, 4}^{\mathrm{u}}$ is constant and equal to $60 \mathrm{~km} / \mathrm{h}$, as well as the slope angle $x_{i, k, 5}^{\mathrm{u}}=0$. The control input $u_{i, k}$ impacts the heading angle $x_{i, k, 6}^{\mathrm{u}}$. The UAV state vector $\mathbf{x}_{i, k}^{\mathrm{u}}$ evolves according to

$$
\left(\begin{array}{c}
x_{i, k+1,1}^{\mathrm{u}} \\
x_{i, k+1,2}^{\mathrm{u}} \\
x_{i, k+1,3}^{\mathrm{u}} \\
x_{i, k+1,4}^{\mathrm{u}} \\
x_{i, k+1,5}^{\mathrm{u}} \\
x_{i, k+1,6}^{\mathrm{u}}
\end{array}\right)=\left(\begin{array}{c}
x_{i, k, 1}^{\mathrm{u}}+T x_{i, k, 4}^{\mathrm{u}} \cos \left(x_{i, k, 5}\right) \cos \left(x_{i, k, 6}^{\mathrm{u}}+u_{i, k}\right) \\
x_{i, k, 2}^{\mathrm{u}}+T x_{i, k, 4}^{\mathrm{u}} \cos \left(x_{i, k, 5}\right) \sin \left(x_{i, k, 6}^{\mathrm{u}}+u_{i, k}\right) \\
x_{i, k, 3}^{\mathrm{u}}+T x_{i, k, 4}^{\mathrm{u}} \sin \left(x_{i, k, 5}\right) \\
x_{i, k, 4}^{\mathrm{u}} \\
x_{i, k, 5}^{\mathrm{u}} \\
x_{i, k, 6}^{\mathrm{u}}+u_{i, k}
\end{array}\right) .
$$

The parameters of the cost function (19) of the UAVs are set to $\alpha^{\mathrm{m}}=1.3, \alpha^{\mathrm{d}}=4.5, \alpha^{\mathrm{s}}=3500, d^{\mathrm{col}}=5, \alpha^{\mathrm{s} 1}=10$, $\alpha^{\mathrm{s} 2}=3$. The communication range is unlimited but the communication is impossible when an obstacle intersects the line of sight of two UAVs. No delay is considered and the communication period is equal to the sampling period. The prediction horizon for the MPC is $h=2$. The UAVs are equipped with identical optical sensors able to detect targets within their FoV. The sensor opening angles are equal to $\pi / 4$ in both azimuth and elevation. As the targets are assumed to move on the $z=0$ plane, when a target is detected at time $k$, the measurement equation (5) provides a noisy measurement $\mathbf{y}_{i, j, k}$ of $\left(x_{j, k, 1}^{\mathrm{t}}, x_{j, k, 2}^{\mathrm{t}}\right)^{\top}$ with an additive noise $\mathbf{w}_{i, j, k}$ bounded in $[-5 \mathrm{~m}, 5 \mathrm{~m}]$. 
$N_{\mathrm{f}}=2$ false targets moving in a plane are also considered with $\mathbf{x}_{\ell, 0}^{\mathrm{f}} \in \mathbb{R}^{4}$. The dynamics of false targets are the same as that of true targets. A false target with state $\mathbf{x}_{\ell}^{\mathrm{f}}$ is confused with a true target when it is observed by some UAV $i$ with state $\mathbf{x}_{i}^{\mathrm{u}}$ satisfying (4), where

$g_{i, \ell}\left(\mathbf{x}_{i}^{\mathrm{u}}, \mathbf{x}_{\ell}^{\mathrm{f}}\right)=\left(\left(\mathbf{x}_{i}^{\mathrm{u}}-\mathbf{x}_{\ell}^{\mathrm{f}}\right) \cdot \mathbf{a}_{\ell}\right)^{2}-\left(\mathbf{x}_{i}^{\mathrm{u}}-\mathbf{x}_{\ell}^{\mathrm{f}}\right)^{2} \mathbf{a}_{\ell}^{2}\left(\cos \lambda_{\ell}\right)^{2}$

represents a half circular cone of $\mathbb{R}^{5}$ with the aperture $2 \lambda_{\ell}$ uniformly distributed within $[\pi / 15, \pi / 5]$. The cone vertex is $\mathbf{x}_{\ell}^{\mathrm{f}}$ and its axis is parallel to

$$
\mathbf{a}_{\ell}=\left(\sin \gamma_{\ell} \cdot \cos \beta_{\ell}, \sin \gamma_{\ell} \cdot \sin \beta_{\ell}, \cos \gamma_{\ell}, 0,0,0\right)^{\top},
$$

where the azimuth $\beta_{\ell}$ is bounded in $[0,2 \pi]$ and the elevation angle $\gamma_{\ell}$ is equal to the aperture.

The search area is a square of $500 \times 500 \mathrm{~m}^{2}, N_{\mathrm{o}}=5$ obstacles are randomly placed within the search area. The obstacles are modeled as rectangular cuboids with a constant height of $100 \mathrm{~m}$ while their lengths and widths are chosen at random in the interval $[30 \mathrm{~m}, 70 \mathrm{~m}]$.

Different approximate representations of a set can be considered, e.g., ellipsoids, polytopes, or interval vectors. For most simple linear dynamics and states in $\mathbb{R}^{2}$, a set may be represented as unions of $2 \mathrm{D}$ polygons, e.g., using the MATLAB toolbox Polyshape. In the case of nonlinear dynamics or higher dimensional state vectors, unions of interval vectors (subpavings) are better suited [11]. The direct and inverse image of a subpaving by a function my be computed using tools from interval analysis, e.g., the INTLAB library [20].

Here polyshapes have been used. To apply the target dynamics (2) in (8) and (9) to a set, we evaluate the maximal possible traveled distance of the target and inflate the set by this distance.

The simulation has been launched on a Windows 10 machine with an Intel Xeon W-2123 processor. It uses the MATLAB R2019a. A simulation with a length of $400 \mathrm{~s}$ took $2700 \mathrm{~s}$ in average.

Fig. 4 shows a typical evolution of the projection of the sets $\mathbb{X}_{1, k}$ and $\overline{\mathbb{X}}_{1, k}$ on $\mathbb{R}^{2}$ evaluated by UAV $i=1$ (in red). Initially, $\overline{\mathbb{X}}_{1, k}$ is large $(k=72)$. One observes at time $k=$ 132 that a false target has been considered as a true target. Measurements coming from an other UAV at time $k=137$ help to eliminate this false target from further consideration. When $k$ is sufficiently large, $\overline{\mathbb{X}}_{1, k}$ becomes empty and $\mathbb{X}_{1, k}$ contains all possible state values of the targets.

Fig. 5 shows the projection of $\mathbb{X}_{1, k}$ and $\overline{\mathbb{X}}_{1, k}$ on $\mathbb{R}^{2}$ evaluated by UAV $i=1$ (in red) for two consecutive time steps $(k=17$ and $k=18$ ). The figure highlights the situation when disconnected communication graphs are reconnected. UAV $i=1$ receives information from remote neighbors at time $k=18$ and can update its estimate $\overline{\mathbb{X}}_{1, k}$. It only receives the information from its direct neighbors.Videos illustrating this situation of sudden reduction of $\overline{\mathbb{X}}_{i, k}$, when UAVs previously unable to communicate, are again communicating can be accessed at https://drive.google.com/open? id=1 HQO0 ny zVd1 nqMmXPA3Vmu1d5QCGUgUS-
Fig. 6 shows the mean value over 100 simulations of $\phi\left(\mathbb{X}_{k}\right) / \phi\left(\mathbb{X}_{0}\right)=\frac{1}{N_{\mathrm{u}}} \sum_{i=1}^{N_{\mathrm{u}}} \phi\left(\mathbb{X}_{i, k}\right) / \phi\left(\mathbb{X}_{0}\right)$ and $\phi\left(\overline{\mathbb{X}}_{k}\right) / \phi\left(\mathbb{X}_{0}\right)=\frac{1}{N_{\mathrm{u}}} \sum_{i=1}^{N_{\mathrm{u}}} \phi\left(\overline{\mathbb{X}}_{i, k}\right) / \phi\left(\mathbb{X}_{0}\right)$, which are components of $\Phi\left(\mathbb{X}_{k}, \overline{\mathbb{X}}_{i, k}\right)$ in (17). In this application the measure $\phi(\mathbb{X})$ is defined as the surface of the projection of $\mathbb{X}$ on the plane in which targets are evolving. Fig. 6 shows that $\phi\left(\overline{\mathbb{X}}_{k}\right)$ converges towards 0 and is already very close to zero at step $k=300$. Due to $\alpha^{\mathrm{m}}=1.3$ in (20), the UAVs try to reduce in priority $\phi\left(\overline{\mathbb{X}}_{k}\right)$ compared to $\phi\left(\mathbb{X}_{k}\right)$. The measure of the target state estimation uncertainty $\phi\left(\mathbb{X}_{k}\right)$ starts increasing, due to the discovery of new targets, and to the fact that at the beginning, UAVs favor exploration of unexplored areas to discover new targets. In a second phase, when the search space has been largely explored, UAVs try to reduce the sate estimation uncertainty $\phi\left(\mathbb{X}_{k}\right)$. At time $k=400, \phi\left(\mathbb{X}_{k}\right) / \phi\left(\mathbb{X}_{0}\right)=0.035$ in average, which corresponds to an area of $65 \times 65 \mathrm{~m}^{2}$ in average.

Fig. 7 shows the trajectory of a UAV trying to get a more accurate estimate of the state of an already detected target. The figure illustrates the different trajectories adopted in the presence and absence of obstacles.

\section{Conclusions}

This paper addresses the problem of multi-target search and tracking using a fleet of drones in the presence of distracting false targets and obstacles. A set-membership estimation algorithm is developed to process the information collected by the UAVs. Each UAV evaluates three different sets: one set guaranteed to contain the state of already detected targets, one set to which not yet detected targets may belong to, and one set proven not to contain any target.

The originality of the proposed method is that it does not require labeling of the targets, and thus the collected observations do not require being linked to a specific target. To increase the information available on the target locations, a cost function is developed which leads the UAVs to the not yet explored set and prevents the UAVs from colliding with obstacles. Each UAV determines its trajectory using the information it has collected and the one provided by its communicating neighbors.

The distributed evaluation of control inputs relies on a model predictive control approach. The simulations illustrate the robustness of the proposed approach. All targets in the search area are detected and the estimation uncertainty gradually decreases. Some subsets of the target state estimates might originate from the presence of false targets. Nevertheless, these subsets are reduced during the simulations thanks to new observations from different points of view.

Further extensions of this paper include the presence of both false targets and non-observation of true targets at the same time. Additionally we will consider the impact on the control input design of limited UAV resources.

\section{Acknowledgments}

The authors gratefully thank the french Agence de l'Innovation de Défense (AID) for its fundings. 

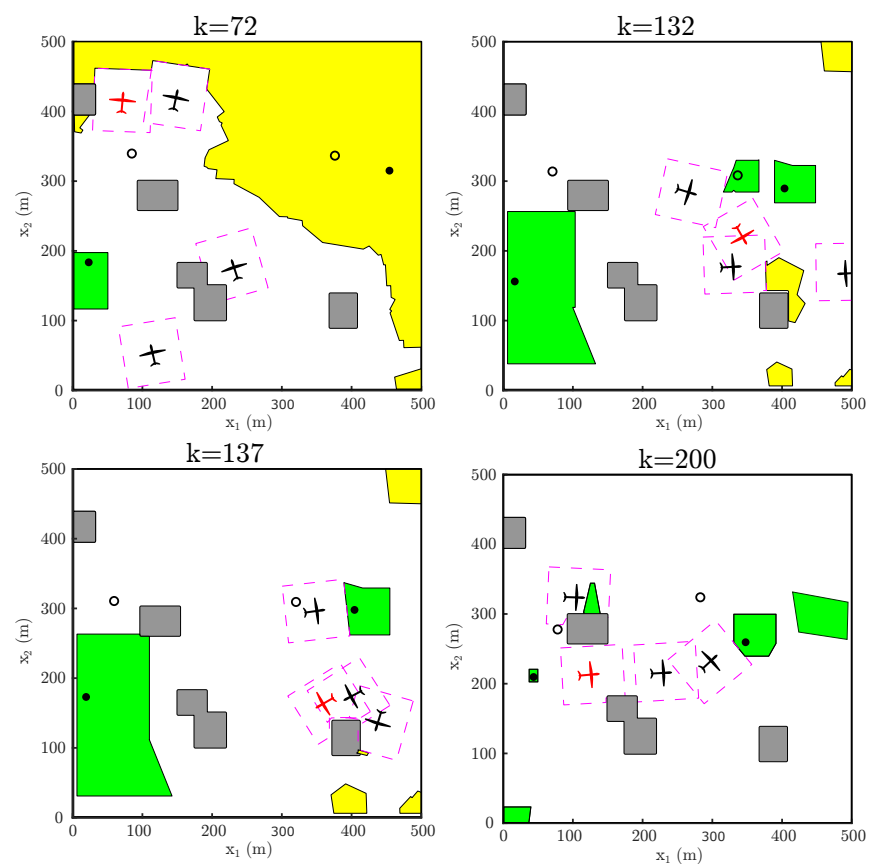

Fig. 4. Projection of $\mathbb{X}_{1, k}$ (green) and $\overline{\mathbb{X}}_{1, k}$ (yellow) on $\mathbb{R}^{2}$ evaluated by UAV $i=1$ (red) for different time steps. Simulation with two true targets (filled circles) and two false targets (empty circles). The FoVs are magenta.
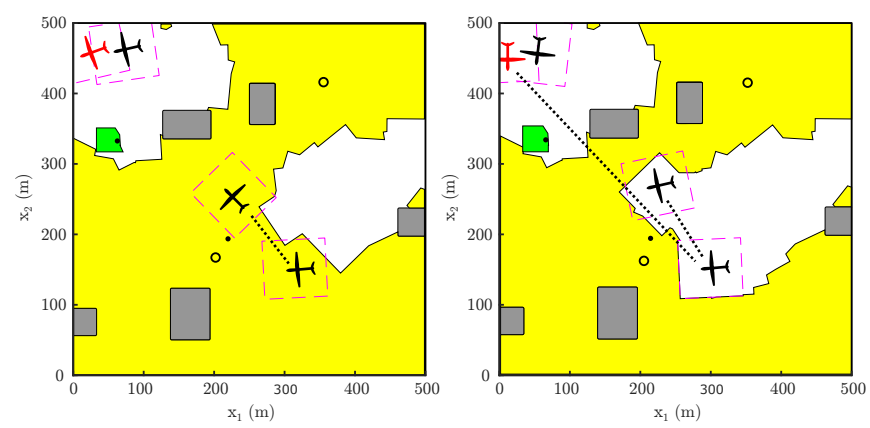

Fig. 5. Projection of $\mathbb{X}_{1, k}$ and $\overline{\mathbb{X}}_{1, k}$ on $\mathbb{R}^{2}$ evaluated by UAV $i=1$ (red) for $k=17$ (left) and $k=18$ (right); At $k=17$, UAV 1 is unable to communicate with one of its distant neighbors due to an obstacle; this communication is again possible at $k=18$ and leads to a significant reduction of the size of $\overline{\mathbb{X}}_{1, k}$. The dotted lines indicate the connected UAVs.

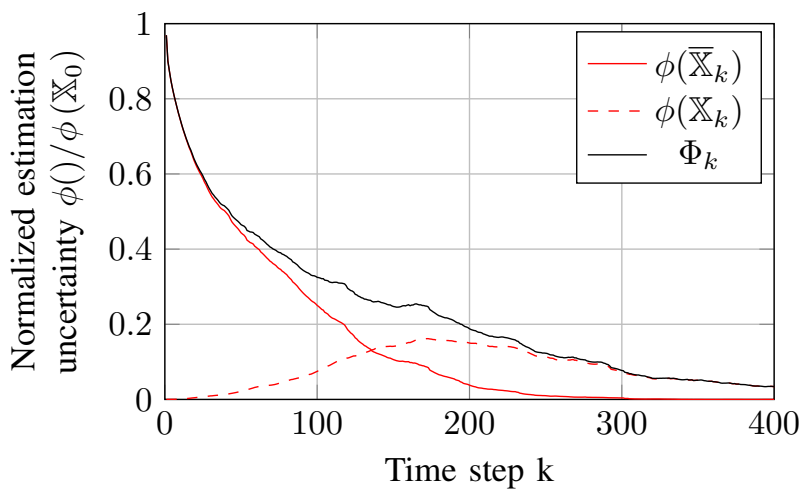

Fig. 6. Mean value over 100 simulations of $\phi\left(\mathbb{X}_{k}\right) / \phi\left(\mathbb{X}_{0}\right)$ (relative size of the target state set estimate), $\phi\left(\overline{\mathbb{X}}_{k}\right) / \phi\left(\mathbb{X}_{0}\right)$ (relative size of the set still to explore), and $\Phi_{k} / \phi\left(\mathbb{X}_{0}\right)$ (relative set estimation uncertainty).
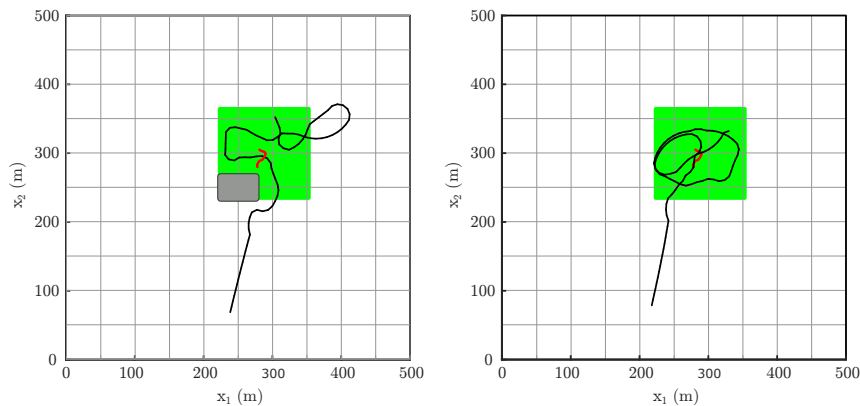

Fig. 7. UAV trajectory (black line) in presence of an obstacle (right) and without obstacles (left); the target trajectory is in red and the set estimate $\mathbb{X}_{i, k}$ in green.

\section{REFERENCES}

[1] Y. Bar-Shalom, P. Willett, and X. Tian. Tracking and data fusion. YBS publishing Storrs, USA, 2011.

[2] J. Blinn. Me and my (fake) shadow. IEEE Comput. Graph. Appl., $8(1): 82-86,1988$

[3] E Camacho and C. Alba. Model predictive control. Springer Science \& Business Media, 2013

[4] V. Drevelle, L. Jaulin, and B. Zerr. Guaranteed characterization of the explored space of a mobile robot by using subpavings. In Proc. IFAC NOLCOS, pages 44-49, 2013

[5] J. Foraker, J. O. Royset, and I. Kaminer. Search-trajectory optimization: Part I, formulation and theory. J. Optim. Theory. Appl., 169(2):530-549, 2016

[6] J. Foraker, J. O. Royset, and I. Kaminer. Search-trajectory optimization: Part II, algorithms and computations. J. Optim. Theory. Appl., 169(2):550-567, 2016.

[7] F. Gu, Y. He, and J. Han. Active persistent localization of a three-dimensional moving target under set-membership uncertainty description through cooperation of multiple mobile robots. IEEE Trans. Ind. Electron., 62(8):4958-4971, 2015.

[8] S. He, H.-S. Shin, and A. Tsourdos. Constrained multiple model bayesian filtering for target tracking in cluttered environment. IFACPapersOnLine, 50(1):425-430, 2017.

[9] J. Hu, L. Xie, J. Xu, and Z. Xu. Multi-agent cooperative target search. Sensors (Basel), 14(6):9408-9428, 2014.

[10] J. Ibenthal, L. Meyer, M. Kieffer, and H. Piet-Lahanier. Bounded-error target localization and tracking in presence of decoys using a fleet of uavs. In Proc. IFAC World Congress 2020, To appear.

[11] Michel Kieffer, Isabelle Braems, Eric Walter, and Luc Jaulin. Guaranteed set computation with subpavings. In Scientific Computing, Validated Numerics, Interval Methods, pages 167-178. Springer, 2001.

[12] P. Li and H. Duan. A potential game approach to multiple uav cooperative search and surveillance. Aerosp. Sci. Technol., 68:403415, 2017.

[13] Jason R Marden and Jeff S Shamma. Revisiting log-linear learning: Asynchrony, completeness and payoff-based implementation. Games and Economic Behavior, 75(2):788-808, 2012.

[14] M. Morari and J. H. Lee. Model predictive control: past, present and future. Comput. Chem. Eng., 23:667-682, 1999.

[15] Reza Olfati-Saber. Distributed kalman filter with embedded consensus filters. In Proc. IEEE CDC, pages 8179-8184. IEEE, 2005

[16] M. Raap, M. Preuß, and S. Meyer-Nieberg. Moving target search optimization-a literature review. Comput. Oper. Res., 105:132-140, 2019.

[17] L. Reboul, M. Kieffer, H. Piet-Lahanier, and S. Reynaud. Cooperative guidance of a fleet of uavs for multi-target discovery and tracking in presence of obstacles using a set membership approach. In Proc. IFAC ACA, pages 340-345, 2019.

[18] S. Reynaud, M. Kieffer, H. Piet-Lahanier, and L. Reboul. A setmembership approach to find and track multiple targets using a fleet of uavs. In Proc. IEEE CDC, pages 484-489, 2018.

[19] Y. Rochefort, H. Piet-Lahanier, S. Bertrand, D. Beauvois, and D. Dumur. Model predictive control of cooperative vehicles using systematic search approach. Control. Eng. Pract., 32:204-217, 2014.

[20] S. M. Rump. Intlab-interval laboratory. In Developments in reliable computing, pages 77-104. Springer, 1999. 